\title{
Implementation of a Healthy Food and Beverage Policy at a Public University
}

\author{
Zachary Rickrode-Fernandez, $\mathrm{MPH}^{1,2}$; Janice Kao, $\mathrm{MPH}^{3}$; Mary N.R. Lesser, PhD, $\mathrm{RD}^{4,5,6}$; \\ Kim Guess, BS, RD ${ }^{7}$
}

\begin{abstract}
University nutrition policies are a useful step toward improving the food environment for students, faculty, and staff, leading to improved health outcomes for the campus community. As 1 of the first universities to adopt and implement a campus-wide nutrition policy, the objective of this report is to share the university's experience with policy development, implementation, monitoring and evaluation, challenges, facilitators, and recommendations to inform these processes for future university nutrition policies.
\end{abstract}

Key Words: nutrition policy, university, vending, dining, sugar-sweetened beverages (J Nutr Educ Behav. 2021;53:891-899.)

Accepted June 16, 2021. Published online August 6, 2021.

\section{INTRODUCTION}

Across the nation, nearly 22 million students are enrolled in college or graduate school, ${ }^{1}$ and more than 4 million people are employed by a postsecondary institution. ${ }^{2}$ For the millions of people who spend their days working and learning on college campuses, where they consume countless meals, snacks, and beverages, the quality and healthfulness of the foods and beverages available are important. Higher education institutions are working to provide opportunities for health and wellness for campus communities. Many of these institutions participate in the Healthier Campus Initiative through the Partnership for a Healthier America ${ }^{3}$ or have their own guidelines that focus on specific aspects of the food environment, such as increasing healthy food/ beverage offerings. The University of California Berkeley, referred to as the university or UC Berkeley hereafter, was 1 of the first universities in the nation to enact a campus-wide nutrition policy. The following report describes the process of developing and implementing the Food and Beverage Choices (FBC) policy, ${ }^{4}$ as well as some key lessons learned.

\section{Background and Overview}

In 2015, the vice-chancellor of administration and finance charged the UC Berkeley University Health Services (UHS) with developing a nutrition policy to ensure that healthy food and beverage options on campus were accessible, affordable, and appealing. Because of the increased privatization of the campus food environment,

\footnotetext{
${ }^{1}$ Center for Environmental Health, Oakland, CA

${ }^{2}$ School of Public Health, University of California Berkeley, Berkeley, CA

${ }^{3}$ University of California, Division of Agriculture and Natural Resources, Nutrition Policy Institute, Oakland, CA

${ }^{4}$ Department of Nutritional Sciences and Toxicology, University of California Berkeley, Berkeley, CA

${ }^{5}$ University of California San Francisco Benioff Children's Hospital Oakland, Oakland, CA

${ }^{6}$ Children's Hospital Oakland Research Institute, Oakland, CA

${ }^{7}$ University Health Services, University of California Berkeley, Berkeley, CA

Conflict of Interest Disclosure: The authors have not stated any conflicts of interest.

Address for correspondence: Kim Guess, BS, RD, University Health Services, University of California Berkeley, 2222 Bancroft Way, Berkeley, CA 94720; E-mail: kguess@berkeley.edu (C) 2021 The Authors. Published by Elsevier Inc. on behalf of Society for Nutrition Education and Behavior. This is an open access article under the CC BY-NC-ND license (http:// creativecommons.org/licenses/by-nc-nd/4.0/)

https://doi.org/10.1016/j.jneb.2021.06.009
}

university leadership recommended a nutrition policy to help protect against an increase in unhealthy foods and marketing. The goal of the policy was to encourage consumption of healthier foods and beverages by making their selection easier, rather than taking the less healthy food and beverage options away.

The FBC policy established nutrition standards for retail foodservice and markets, vending machines, athletic concessions, dining halls, and university-sponsored meetings. Health education alone-in the absence of changes to the food environment-does not always produce lasting behavioral change. ${ }^{5,6}$ Therefore, the policy ensured that in addition to promoting healthier choices through marketing and advertising standards, there were accessible whole, fresh foods and healthier alternatives to sugar-sweetened beverages present in all university food environments. A summary of select FBC standards is described in Table 1.

\section{Health education alone- in the absence of changes to the food environment-does not always produce lasting behavioral change.}

The FBC policy supported the creation of healthier food environments, which is 1 approach to improve the 
Table 1. University of California Berkeley Food and Beverage Choices Policy: Summary of Select Standards ${ }^{4}$

Food standards

- Entrees and sides should include options with fresh vegetables and fruit, $100 \%$ whole grains, plant-based protein, and lean meats (such as skinless chicken breast)

- No trans-fats

- Offer small-sized (eg, <2 oz) options for baked goods/desserts where these are sold

- Use healthier cooking techniques such as baking, roasting, stir-fry, grilling, and poaching

- At least half of all snacks, entrees, and meals (respectively) sold in vending machines must meet the following per-package standards:

- $\leq 250 / 400 / 700$ calories

- $\leq 35 \%$ of total calories from fat

- $\leq 10 \%$ of total calories from saturated fat

- $\leq 20 / 15 / 35$ grams of total sugar

- $\leq 360 / 600 / 1000$ milligrams of sodium

- At least 1 snack vending machine option is $\leq 100$ calories

- The first ingredient listed must be a fruit, vegetable, low-fat dairy, protein, or whole grain

Beverage standards

- Healthier beverages include water, diet, and low-calorie beverages with $\leq 40$ calories per $8 \mathrm{fl}$ oz, unsweetened coffee and tea, $100 \%$ fruit or vegetable juice and juice blends, unflavored and unsweetened $1 \%$ or nonfat milk, and unflavored, unsweetened nondairy milk, such as soy or almond milk

- Healthier beverages must occupy at least $70 \%$ of shelf space in retail settings and visible selection buttons in vending machines

- Packaged energy drinks and energy-type beverages with caffeine as an additive are limited to $71 \mathrm{mg}$ of caffeine per $12 \mathrm{fl}$ oz

- Maximum size of 20 oz container for sugar-sweetened beverages (all drinks with added sugar exceeding 40 calories per fl oz)

- Maximum size of $21 \mathrm{fl} \mathrm{oz} \mathrm{for} \mathrm{fountain} \mathrm{drinks,} \mathrm{preferably} 16 \mathrm{fl} \mathrm{oz}$

Guidelines for healthier meetings and events

- Food and beverages purchased with university funds for university meetings and events should include healthy options that meet the standards listed above

- Water must be offered as a beverage choice, preferably in bulk, to minimize the use of single-use plastic bottles

Dining commons

- At lunch and dinner, offer a salad bar and lean meat when meat is served

- Offer at least 1 cooked vegetable at breakfast and 2 at lunch and dinner

- Offer fruit, a minimum of 2 whole-grain options, and a minimum of 1 plant-based protein at all meals

- Limit deep-fried options to 2 per meal

\section{Marketing and advertising standards}

- Advertising and promotion of specific products should prioritize healthier foods/drinks that meet the standards listed above (eg, identify healthy products with an icon or logo)

- Healthy foods and beverages should be placed in more prominent or best-selling positions and priced equal to or less than similar less healthy items

\section{Coffee and tea providers under university agreements}

- The vendor should post calorie counts on menus and provide information on the added sugar content of beverages

- The vendor will display a healthier beverage menu

dietary behavior of the campus popu-

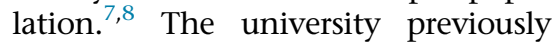
adopted complementary campus wellness policies that are focused on breastfeeding, food safety, food insecurity, sustainability, alcohol, and physical activity. ${ }^{9}$

\section{DESCRIPTION OF THE INTERVENTION}

A simplified timeline of the policy process is depicted in the Figure. Key tasks and partners involved in policy development, preimplementation, implementation, monitoring, and evaluation are listed in Table 2 and summarized below.

\section{Development}

After several years of consultation and negotiation with stakeholders associated with the university, the FBC policy was issued on January 1, 2018 and officially went into effect on January 1, 2019. The policy included planned updates at least every 5 years.
The Nutrition Policy Working Group led the development of the policy and reviewed existing nutrition policies and recommendations to create an initial draft of standards for implementation at the university. The working group modeled nutrition standards on a variety of guidelines, primarily the 2015 Dietary Guidelines for Americans ${ }^{10}$ and the Culinary Institute of America's Menus of Change. ${ }^{11}$ In addition, they included a standard regarding highly caffeinated drinks, which are often mixed with alcohol, to address the adverse health outcomes 


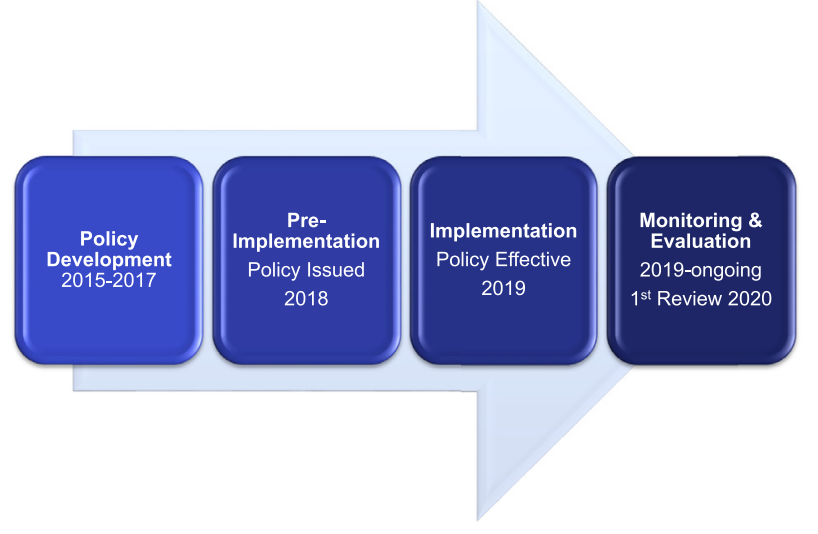

Figure. A simplified timeline of the University of California Berkeley Food and Beverage Choices policy process.

frequently seen in the university urgent care clinic. The policy set a maximum allowable added caffeine content of $71 \mathrm{mg}$ per $12 \mathrm{fl} \mathrm{oz}$ in accordance with the generally recognized as safe limit for caffeine in soda as determined by the US Food and Drug Administration. ${ }^{12}$

The group engaged a wide variety of campus stakeholders in the review process to obtain buy-in and ensure that the policy would be both viable and impactful. Stakeholders included members of the university administration, students, faculty, account managers, and vendors. The working group presented drafts of the policy through meetings, emails, and inperson presentations to give each stakeholder group an opportunity to provide feedback. Among the input received were concerns around affordability, the potential impact on sales, sustainability, waste, and how to incentivize the purchase of healthier food. The final version of the policy addressed these concerns.

\section{Preimplementation}

Once the university officially issued the FBC policy, campus food and beverage entities had 1 year to make any changes needed to be in policy compliance. This phase will be referred to as preimplementation.

The Centers for Disease Control and Prevention's Improving the Food Environment Through Nutrition Standards guide ${ }^{13}$ recommends minimal staffing of a policy coordinator, project coordinator, and registered dietitian during the first year of implementation. At UC Berkeley, the wellness dietitian served all 3 of these roles $(10-20 \mathrm{~h} / \mathrm{wk})$ and had primary responsibility for policy implementation. The UC Global Food Initiative also funded a graduate student fellow to assist with the policy for 1 year ( $3 \mathrm{~h} / \mathrm{wk})$. The dietitian and the graduate student fellow, from here on referred to as the policy team, completed much of the preimplementation and initial implementation work. In addition to the policy team, a student union nutrition intern $(10 \mathrm{~h} / \mathrm{wk})$ and an undergraduate community nutrition class played key roles in policy preimplementation, implementation, and evaluation. The costs of policy implementation included the salary for the dietitian, intern, and graduate student fellow and marketing materials such as handouts and other signage.

A primary goal during preimplementation was to collect baseline data on aspects of the prepolicy food and beverage environment, including observations of what foods and beverages were being sold, how they were displayed, and customer perceptions of the food environment.

To measure the change in perception of the food and beverage offerings and desire for healthier options on campus among students, staff, faculty, and guests, the policy team created a self-administered intercept survey, referred to here as the Food Environment Survey. The University of California Berkeley Institutional Review Board determined that the data collected and presented in this report were not considered human subjects research, as stated in a written document that the authors have on file and is available upon request. The policy team trained an undergraduate community nutrition class to collect responses by approaching people near campus restaurants or vending machines and inviting them to complete the questionnaire. The goal was to collect data from a convenience sample of individuals representing a variety of campus affiliations. The students collected 273 responses from 224 undergraduate students, 20 graduate students, 12 staff, and 17 visitors. The results are discussed in a later section. The survey findings were used to demonstrate the demand for healthier food and beverages and were shared in policy education materials, such as a presentation for campus restaurant managers.

The policy team also trained the same nutrition class to perform audits of each university-operated food and beverage vending machine using vending audit surveys developed by UHS and programmed into an online survey platform. For each vending machine, students counted and recorded the number of items that met the policy standards to determine the percentage of healthier options.

To conduct restaurant menu audits, the policy team visited campus restaurants and retail locations that were required to comply with the policy at random hours. The menu assessment (see Supplementary Data) was developed by 2 graduate students and was on the basis of a model by the Philadelphia Department of Public Health. ${ }^{14}$

In addition, the policy team met with owners, managers, and staff of each location or retail group to educate everyone about the policy, identify which areas would need improvement, and offer suggestions 
Table 2. Key Tasks and Partners for Policy Development, Implementation, and Evaluation

\section{Key Tasks}

\section{Development}

1. Committee formed to lead policy development, known as the

2. The working group reviewed national nutrition policies, best practices, public health guidelines, and toolkits and consulted with subject matter experts throughout the country

3. The working group drafted nutrition standards and shared them with stakeholder groups

4. Stakeholders reviewed standards and provided feedback

5. The working group revised standards on the basis of stakeholder feedback

6. Policy submitted to university policy review committee for official policy adoption Nutrition Policy Working Group

\section{Key Partners}

Nutrition Policy Working Group: staff/faculty from University Health Services, School of Public Health, Department of Nutritional Sciences and Toxicology, and Berkeley Food Institute Stakeholder review process:

- Staff from campus dining, concessions, student services, student union, business contracts and partnerships, sustainability, health inspection, athletics, basic needs, campus legal, worksite wellness

- Faculty from the departments of law, journalism, education, nutritional sciences, public policy, economics, psychology, environmental science

- Students from the student union, residence hall assembly, graduate assembly, public health, and community nutrition classes

Food and beverage vendors: campus-wide beverage contract, snack vending, coffee/tea provider, campus restaurants

\section{Preimplementation}

1. Formed the policy team to carry out preimplementation and implementation

2. The policy team developed baseline data collection forms:

- Menu and vending machine audits

- Survey on perceptions of food environment

3. Students trained to conduct baseline assessments:

- Conducted surveys with students, staff, visitors outside of campus restaurants and vending machines

- Observed foods and beverages offered in vending machines and campus restaurants/retail locations

4. The policy team introduced policy and made sure that campus restaurants/retail were aware of the upcoming policy

\section{Implementation}

1. The policy team promoted the policy widely via campus messaging, social media posts, and a dedicated policy webpage

2. Partnered with other campus groups to help promote and support policy implementation

3. The policy team invited campus experts to serve on a policy advisory committee

4. The policy team met with retail managers and vendors to review baseline audit results and confirm progress with meeting standards

5. The policy team conducted policy training with other campus entities that serve food (eg, event planners)

6. The advisory committee met quarterly to review policy implementation progress and brainstorm strategies

\section{Monitoring and evaluation}

1. Collect data to assess implementation progress and change over time:

- Yearly audits of foods/beverages offered in restaurants, retail, and vending machines

- Sales data from restaurants, retail, and vending

2. Repeat the Food Environment Survey to assess change in perceptions of healthy food availability and desire for healthy food and beverages

3. Review and revise policy language to strengthen and clarify standards, include new campus food/beverage entities, etc
Policy team: University Health Services wellness program dietitian (lead) and a funded graduate student fellow, recruited specifically to help with policy implementation Baseline data collection team:

- Policy team

- Undergraduate nutrition class

Nutrition Policy Advisory Committee: representatives from university wellness program and health promotion, social services dietitians, dining services, the nutrition department, Office of Environment, Health and Safety, Berkeley Food Institute, UC Global Food Initiative, UC Nutrition Policy Institute, the Student Union, and the Student Nutrition Advocacy Club

Campus partners: sustainability/zero waste, Coalition for

- Policy team

- Nutrition Policy Advisory Committee

- Undergraduate nutrition class

- Vendors to provide sales data Healthy Campus Food and Beverages 
on how to fully comply with the new standards. The policy team provided a presentation, vendor guide, frequently asked questions webpage, and samples of advertising materials, such as register signs and window clings.

\section{Implementation}

Once the FBC policy took effect, the policy team launched a policy promotion campaign using several platforms to raise awareness of the policy, including a policy webpage with a promotional video, ${ }^{15}$ campus announcements, and social media posts. The policy team also invited staff and faculty with expertise related to the implementation and evaluation of the FBC policy to serve on an advisory committee. The Nutrition Policy Advisory Committee (NPAC) meets quarterly to review and discuss policy implementation progress and strategy. See Table 2 for a list of committee members. Implementation experiences varied widely by type of retail entity and are described below.

University-operated foodservice. The 5 university-run restaurants were not yet in compliance with the policy effective date. The coronavirus disease 2019 (COVID-19) pandemic further delayed compliance and caused all but 1 restaurant to close temporarily. Before closing, most restaurants were close to meeting all standards except for the healthier beverage standards. The lack of compliance may be attributed to turn over in leadership, having a pouring rights contract, and a shift in priorities because of the COVID-19 pandemic. The dining halls were added to the FBC policy in the first revision but have not yet been audited because of pandemic-related service restrictions. However, on the basis of information provided by dining management, $93 \%$ of the menu options in the dining halls met the nutrition standards.

Food and beverage vending. The policy team and the snack vending company agreed on a standard schematic for all machines that reflect the $50 \%$ minimum requirement of healthier products. As part of the implementation process, the policy team scheduled regular meetings with vendor representatives to monitor compliance, review vending sales, and introduce new products.

A single beverage corporation currently has an exclusive pouring rights contract across student and athletic services. Because the contract existed before the policy was issued, the corporation is not required to comply until the contract is renewed or a new contract is signed. Following the vending audit, the policy team presented the audit results and sampled policy-compliant vending machine schematics to the corporation's campus liaison for implementation in the summer of 2019. Although the beverage corporation did not initially make updates to their beverage vending options, they began replacing some soda machines with 100\% healthier beverage machines and increasing the beverage mix to at least $70 \%$ healthier in all other machines in the fall of 2020 .

Student union. The student union houses a variety of food and beverage vendors, many of which signed leases before the policy was approved. During implementation, the student union employed an undergraduate nutrition intern to support the FBC policy and ensure student satisfaction with food service in the student union. In the Fall of 2019, 1 coffee shop and 3 of 5 eateries were in compliance with the policy. All foodservice locations closed because of the COVID-19 pandemic, and several locations closed permanently when their leases expired.

Coffee and tea vendors. The university partnered with a coffee/tea provider which has several locations on campus. Under the FBC policy, this provider was required to work with the university to address added sugar. This included posting calories on the menu, in accordance with the 2018 US Food and Drug Administration menu labeling rule, promoting point-of-service information on added sugar content for coffee/tea beverages, and creating a healthier beverage menu. The healthier beverage menu was completed and posted in January, 2019. In addition, the wellness program dietitian calculated added sugar information for each beverage. This information is available online and in stores.

Athletic concessions. Unique challenges with concessions included infrequent dates of service, customer expectations for traditional concessions (eg, hot dogs, fries, etc), and vendor turnover. Meetings that reviewed the policy and discussed potential marketing ideas to promote healthy options and improve sales have not yet led to any implemented changes, and no progress has been made because of the impact of the COVID-19 pandemic on university athletics. Some of the ideas discussed included creating advertising banners for the healthier options, placing healthier items in prominent locations on the menu, and advertising the policy throughout the stadium, such as on TV screens.

Meetings and events. The policy states that nutritious and sustainable choices must be included when university funds are used to purchase food or beverages for university meetings and events. Water must also be offered as a beverage option. To encourage compliance with this portion of the policy, UHS maintains and promotes a list of caterers that meet certain nutrition standards to campus event planners. The policy is also presented at a yearly event planner certification.

\section{DISCUSSION}

Although the policy took effect on January 1, 2019, implementation is ongoing. For example, nonuniversity-operated foodservice vendors whose leases began before the policy issuance were not required to comply with the policy until renewal of their lease contracts. In addition, some retail entities experienced more implementation challenges than others and have required more time and assistance to become policy compliant. 


\section{Challenges with Implementation}

Complete implementation of and compliance with the policy has varied because of the variety of food and beverage entities across campus and differences in business models, and the types of space and infrastructure available to vendors. The NPAC and policy team identified the following challenges that hindered the successful, complete implementation of the policy.

Communication with vendors. Maintaining consistent communication with different food and beverage operations proved to be the most recurrent difficulty. There were multiple points of contact and the primary form of communication was email, which had a poor response rate. Although more time-consuming, in-person meetings were the most successful method to communicate information, make progress with compliance, and resolve concerns about the policy.

Existing contracts. Because existing contracts and leases could not be modified, several vendors were not yet required to comply with the policy and will not be until new contracts are negotiated. The policy encouraged vendors to voluntarily comply with the FBC standards, but progress has been limited and slow. For example, some beverage vending machines were adjusted to comply with the policy standards in late 2020 , nearly 2 years after the policy went into effect.

Space limitations. Owners of cartbased operations expressed that they had challenges meeting the standards given their lack of storage, shelf space, and infrastructure, in part because of the need to replace items entirely rather than supplement them. The small operating space often means that the food and beverage selection is carefully curated to include only the best-selling products; therefore, there has been resistance in replacing these with newer, healthier items with unknown sales performance.

Procurement. Many vendors purchase products from a supplier, in

which there is less control over portion sizes and what ingredients are used, and are thus limited to the products that are available. For example, current campus suppliers do not provide small- or moderately-sized baked items, pastries, or desserts, making this aspect of the policy a consistent point of noncompliance.

\section{Ninety-five percent agreed that UC Berkeley should have a healthy food environment, and $87 \%$ indicated they would like healthier food and beverage options.}

Monitoring compliance. Compliance monitoring is highly resource intensive, usually requiring in-person observation of what products are sold and how they are displayed. For meetings and events, in particular, there is no mechanism to monitor that healthy options are being offered.

Policy limitations. The policy did not cover all campus food and beverage operations. For example, boba tea shops were not specifically addressed, but by default, they fall short of the healthy beverage standards because of the added sugar content of their drinks. Another major limitation of the policy was the lack of repercussions for noncompliance.

\section{Factors Supporting Implementation}

To address the challenges described above, the policy team has drawn oncampus partnerships and collaborations. Following are some key facilitators of policy implementation.

City and campus culture. The policy maintains widespread support across an array of university stakeholders. The food and health culture of Berkeley, CA, which values organicallygrown food, minimal food processing, and plant-based diets, is reflected in the campus culture. The results of the Food Environment Survey demonstrated a strong demand for healthier options on campus. For example, $95 \%$ of those sampled agreed that UC Berkeley should have a healthy food environment, and $87 \%$ indicated that they would like to have healthier food and beverage options on campus. Restaurant owners and managers also view the standards favorably, and many were already committed to providing healthy food options for customers before the policy.

Student involvement. University students were responsible for a significant portion of the data collected for baseline assessments, a crucial aspect of the preimplementation work. Although initial training of interns and other students can be an investment with something as complex as a university nutrition policy, it is a mutually beneficial and invaluable experiential learning opportunity for undergraduate and graduate students.

Campus collaborations. Partnering with other campus groups and entities like sustainability and zero waste greatly aided policy efforts. Their project support, help with policy promotion, and insights about working with different campus stakeholders or university processes have been invaluable in the development and implementation of the FBC policy. The Berkeley Food Institute, a key campus partner, formed the Coalition for Healthy Campus Food and Beverages ${ }^{16}$ to bring more stakeholders into campus decision-making on FBC. One of the 5 goals was to expand the reach of the FBC policy.

The policy did not appear
to hurt revenues, a
common concern when
implementing nutrition
standards.

\section{DESCRIPTION OF THE EVALUATION}

To evaluate the effectiveness of the FBC policy, multiple types of data are 
regularly collected and analyzed. Vending machine sales data are examined for trends in purchases of healthier and less healthy items, which help inform what strategies are effective for revenue generation and increasing healthy purchasing. Yearly menu reviews and audits of vending machines and retail settings are conducted to track progress with policy compliance. Audits were not conducted in 2020 because of the pandemic. The Food Environment Survey will be repeated to identify any changes in attitudes and perceptions among the campus community regarding food and beverage offerings.

In 2019, the snack vending machine vendor began implementing the new policy, and according to the vending audit, the percentage of stocked healthier snacks increased from 44\% in 2018 (preimplementation) to $49 \%$ in 2019 . Sales reports showed that the percentage of snacks purchased that met the policy standards increased from $27 \%$ in 2018 to $32 \%$ in 2019 . The policy did not negatively affect overall sales as total snack vending sales increased $12 \%$ between 2018 and 2019. Although the beverage vendor is not yet required to comply with the policy, the proportion of vending beverages sold meeting the nutrition standards increased from 33\% to 37\% between 2018 and 2019. The policy also did not negatively impact overall sales as beverage vending sales increased 15\% between 2018 and 2019. Although it is not possible to determine the extent to which these changes were caused by the policy, the policy did not appear to hurt revenues, a common concern when implementing nutrition standards. ${ }^{17}$ Sales in 2020 were severely impacted by the campus closure because of the pandemic, and thus, these data were not included in this analysis.

Other types of financial data have been difficult to obtain. For example, to date, UHS has received limited sales data from campus restaurants, and procurement data as required by the university sustainability policy has been inconsistently reported. Data collection challenges have been further exacerbated by the COVID-19 pandemic, but efforts to streamline the collection of financial data will be pursued in the future.

Although evaluation of student and employee health outcomes would be a helpful marker of policy effectiveness, current funding and resources are insufficient to support a study design that could reasonably assess the policy's impact on health indicators. The university does not currently have a system to collect health data from the campus community, and the original policy plan did not propose a process for gathering these data. The only consistently available health data are from the student health center, which reflects only a small subset of the campus population consisting primarily of students with the student health insurance plan. Without a more robust study design, data on health indicators would be limited in their utility by short follow-up times and a host of confounding factors present in the university environment, among other challenges.

Overall, having multiple types of data for measuring policy success has helped determine what types of training or resources are needed to support successful policy implementation. To encourage compliance, the wellness program dietitian has reached out to the vendors annually to offer support and incentives for policy compliance, such as free promotion through UHS and marketing materials for the vendors to display. The policy team will also continue to promote the policy through social media and other channels to ensure campus awareness and garner interest and support from the campus community.

The creators of the FBC policy have built-in regular opportunities to review and revise the standards to ensure that the policy remains relevant and up-to-date with new dietary guidelines and inclusive of new types of food and beverage vendors that are added to the campus food environment. In the first round of revisions that took effect in November, 2020, the NPAC added dining halls to the list of foodservice operators required to be in compliance with the policy and updated vending machine standards to include vended entrees. The NPAC will notify and work with vendors to make any necessary adjustments to their offerings.

\section{IMPLICATIONS FOR RESEARCH AND PRACTICE}

Overall, UC Berkeley's experience in developing, planning, implementing, monitoring, and evaluating the FBC policy has important implications for future nutrition-related policies on other college campuses. The challenges and factors supporting implementation identified here, as well as additional insights from the university's experience, can inform efforts to make similar policies more feasible, effective, and comprehensive in other higher education food environments. Table 3 provides recommendations for future policy work on the basis of the experiences and lessons learned from the FBC policy.

The FBC policy was well-aligned with the 2012 Institute of Medicine (IOM) report, Accelerating Progress in Obesity Prevention, ${ }^{18}$ which urged the nation's leaders and decisionmakers at all levels to adopt a multisectoral systems approach to slow and even reverse the devastating consequences of poor dietary patterns nationwide. Enacting nutrition policies in higher education settings meets several of the IOM recommendations to make the healthy choice the easy choice, reduce sugar-sweetened beverage consumption, and implement strong nutrition and marketing standards.

In the decade since the publication of these IOM recommendations, improvements to local and federal nutrition policies in kindergarten through 12th-grade education settings have been achieved. A systematic review of the effect of these policies reported that the majority of studies showed improvements in students' diets as access to healthy foods and beverages in the school environment increased. ${ }^{19}$ There is no published literature on higher education nutrition policies; however, a review of nutrition interventions in college settings found positive associations with dietary quality. However, such interventions were short-term and additional research is needed. ${ }^{20}$ 
Table 3. Recommendations for Policy Development, Implementation, Monitoring, and Evaluation

\section{Development}

- Include vendors in policy development discussions to understand implementation concerns and compliance feasibility.

- Ensure the policy would apply to a wide variety of vendors, even vendors that are not currently on campus. Include policy language that applies to vendors not currently on the campus but could be in the future. Ask the vendors about products they may introduce in the coming year.

- Consider including separate specific standards for vendors that do not fit well into existing categories (eg, specialty beverage sellers).

- Think about how to address noncompliance. For example, consider a written enforcement clause, such as denying lease renewals for failure to meet the policy standards or more immediate actions.

- Provide robust definitions for unclear terms, such as lean meats, small portions, and entrees.

Preimplementation and implementation

- Inform the campus community about the policy through channels such as campus webpages, email lists, newsletters, social media, and events. Embed policy information into campus processes (such as catering ordering software) and retail environments (eg, signage in restaurants and stores, clings on vending machines, etc).

- Create vendor educational materials such as an implementation guide, frequently asked questions, and a training presentation. Provide concrete examples of healthier additions or replacements.

- Encourage vendors to "pilot test" new recipes and formulations (eg, vendors can do a test run of smaller-sized baked goods to see how they sell).

- Limit email and phone conversations. Meet with vendors face-to-face to accomplish as many tasks as possible-this includes scheduling the follow-up meeting.

- Work directly with vendors whenever possible for greater effectiveness. If there is a vendor contract manager or other campus liaison, include them in meetings and communications.

- Collaborate with campus decision-makers to ensure they are aware of the policy and how it affects them. Work with the appropriate offices to ensure the policy is included in requests for proposals and leases for food and beverage vendor.

- Partner with other departments that work with vendors, such as the sustainability office, to help vendors comply with multiple university policies.

\section{Monitoring and evaluation}

- Enlist the help of students to audit food and beverage offerings. This can be an experiential learning project for an undergraduate nutrition class.

- Establish periodic review meetings with vendors to share progress and assess areas that need improvement.

- Offer promotional opportunities for vendors as an incentive. For example, promote compliant vendors through social media, webpages, newsletters, and invite them to table (and possibly offer samples) at events.

- Collect data to evaluate the effectiveness of changes made, such as baseline and follow-up food environment data, sales data, and student and/or staff health indicators and behaviors.

University nutrition policies have great potential to ensure that healthy foods and beverages are available where students, staff, and faculty live, work, and learn to facilitate lasting dietary, behavioral changes. Although the effects of nutrition policies have been studied in other settings (eg, kindergarten through 12th-grade schools), more research on the effects of a university-wide nutrition policy is needed. In addition, the FBC policy and other university nutrition policies should be evaluated to understand the impact of such policies on the food environments and health outcomes of campus communities.

\section{ACKNOWLEDGMENTS}

The authors thank the University of California Global Food Initiative provided funding for a graduate fellow to assist with policy implementation. The authors also acknowledge the Berkeley Food Institute, which administered the funding and other support for the graduate fellow and incorporated the Food and Beverage Choices policy into the Coalition for Healthy Campus Food and Beverages; the members of the Nutrition Policy Working Group for drafting the food and beverages choices policy; the undergraduate students of the Nutrition in the Community course for collecting baseline food environment data; the members of the Nutrition Policy Advisory Committee for technical assistance with implementation and policy review; the Associated Students of the University of California Student Union for providing nutrition interns to assist with policy implementation within the student union; and Chrissy Badaracco for her work on the evaluation plan for the UC Berkeley Food and Beverage Choices Policy.

\section{SUPPLEMENTARY DATA}

Supplementary data related to this article can be found at https://doi. org/10.1016/j.jneb.2021.06.009. 


\section{REFERENCES}

1. US Census Bureau's American Community Survey Office. School enrollment: 2019 ACS 1-year estimates. https://data. census.gov/cedsci/table?q=school $\% 20$ en rollment\&tid=ACSST1Y2019.S1401.

Accessed July 12, 2021.

2. National Center for Education Statistics, Integrated Postsecondary Education Data System. Number of people employed by postsecondary institutions. https://nces. ed.gov/ipeds/TrendGenerator/app/ answer/5/30. Accessed May 11, 2021

3. Partnership for a Healthier America. Healthier Campus Initiative: 2019 progress report. https://www.ahealthieramerica.org/progress-reports/2019/ initiatives/healthier-campus-initiative. Accessed March 7, 2021.

4. UC Berkeley University Health Services. Food and Beverage Choices Policy. UC Berkeley University Health Services; 2020. https://campuspol.berkeley. edu/policies/foodbeverage.pdf. Accessed March 7, 2021.

5. Barker M, Kelly MP. Why is changing health-related behaviour so difficult? Public Health. 2016;136:109-116.

6. Johnston CA, Arlinghaus KR. Advocating for behavior change with education. Am J Lifestyle Med. 2017;12:113116.

7. Roy R, Kelly B, Rangan A, AllmanFarinelli M. Food environment interventions to improve the dietary behavior of young adults in tertiary education settings: a systematic literature review. $J$ Acad Nutr Diet. 2015;115:1647-1681.

8. Niebylski ML, Lu T, Campbell N, et al. Healthy food procurement policies and their impact. Int $J$ Environ Res Public Health. 2014;11:2608-2627.

9. UC Berkeley University Health Services. Wellness-related policies and guidelines. https://uhs.berkeley.edu/ facstaff/wellness/healthy-campus/wellness-policies-and-guidelines. Accessed March 7, 2021.

10. US Department of Health and Human Services and US Department of Agriculture. 2015-2020 Dietary Guidelines for Americans. US Department of Health and Human Services and US Department of Agriculture; 2015. https:// health.gov/sites/default/files/2019-09/ 2015-2020_Dietary_Guidelines.pdf. Accessed March 7, 2021.

11. The Culinary Institute of America and Fellows of Harvard College. Principles and resources. https://www.menusofchange.org/principles-and-resources. Accessed March 7, 2021.

12. US Food and Drug Administration. 21CFR-182.1180: food and drugs. https://www.accessdata.fda.gov/scripts/ cdrh/cfdocs/cfcfr/CFRSearch.cfm? $\mathrm{fr}=182.1180$. Accessed March 7, 2021.

13. Blair NA, Gase L, Gunn JP, Lee JM. Improving the Food Environment Through Nutrition Standards; A Guide for Government Procurement. US Department of Health and Human Services, Centers for Disease Control and Prevention, National Center for Chronic Disease Prevention and Health Promotion, Division for Heart Disease and Stroke Prevention; 2011.

14. Get Healthy Philly. Nutrition Standards and Implementation Guide. Philadelphia Department of Public Health; 2018. https://www.phila.gov/media/201810
09160839/Nutrition_Standards_Imple mentation_Guide.pdf. Accessed March 21, 2021

15. UC Berkeley University Health Services. Food and Beverage Choices policy: making healthy options more accessible. https://uhs.berkeley.edu/ foodbeveragepolicy. Accessed March 7, 2021.

16. Berkeley Food Institute. Coalition for healthy campus food and beverages. https://food.berkeley.edu/foodscape/ service-units/coalition-for-healthycampus-food-and-beverages. Accessed March 7, 2021.

17. Viana J, Leonard SA, Kitay B, Ansel D, Angelis P, Slusser W. Healthier vending machines in a university setting: effective and financially sustainable. Appetite. 2018;121:263-267.

18. Committee on Accelerating Progress in Obesity Prevention, Food and Nutrition Board, Institute of Medicine. Glickman D, Parker L, Sim LJ, et al., eds. Accelerating Progress in Obesity Prevention: Solving the Weight of the Nation. National Academies Press; 2012.

19. Mansfield JL, Savaiano DA. Effect of school wellness policies and the Healthy, Hunger-Free Kids Act on food-consumption behaviors of students, 2006-2016: a systematic review. Nutr Rev. 2017;75:533-552.

20. Brace AM, De Andrade FC, Finkelstein B. Assessing the effectiveness of nutrition interventions implemented among US college students to promote healthy behaviors: a systematic review. Nutr Health. 2018;24:171-181. 